\title{
Enhanced current noise correlations in a Coulomb-Majorana device
}

\author{
Hai-Feng Lü, ${ }^{1,2}$ Hai-Zhou Lu, ${ }^{1, *}$ and Shun-Qing Shen ${ }^{3}$ \\ ${ }^{1}$ Department of Physics, South University of Science and Technology of China, Shenzhen 518055, China \\ ${ }^{2}$ Department of Applied Physics, University of Electronic Science and Technology of China, Chengdu 610054, China \\ ${ }^{3}$ Department of Physics, The University of Hong Kong, Pokfulam Road, Hong Kong, China
}

(Received 21 February 2016; revised manuscript received 19 May 2016; published 20 June 2016)

\begin{abstract}
Majorana bound states (MBSs) nested in a topological nanowire are predicted to manifest nonlocal correlations in the presence of a finite energy splitting between the MBSs. However, the signal of the nonlocal correlations has not yet been detected in experiments. A possible reason is that the energy splitting is too weak and seriously affected by many system parameters. Here we investigate the charging energy induced nonlocal correlations in a hybrid device of MBSs and quantum dots. The nanowire that hosts the MBSs is assumed in proximity to a mesoscopic superconducting island with a finite charging energy. Each end of the nanowire is coupled to one lead via a quantum dot with resonant levels. With a floating superconducting island, the devices show a negative differential conductance and giant super-Poissonian shot noise, due to the interplay between the nonlocality of the MBSs and dynamical Coulomb blockade effect. When the island is strongly coupled to a bulk superconductor, the current cross correlations at small lead chemical potentials are negative by tuning the dot energy levels. In contrast, the cross correlation is always positive in a non-Majorana setup. This difference may provide a signature for the existence of the MBSs.
\end{abstract}

DOI: 10.1103/PhysRevB.93.245418

\section{INTRODUCTION}

Quantum transport through topological insulators and superconductors has received a large amount of attention in condensed matter physics over the past few years [1-3]. One of the most influential discoveries is that topological phases supporting Majorana fermions can be realized and engineered in the heterostructures based on $s$-wave superconductors and materials with strong spin-orbit interaction [4-9]. The search for Majorana bound states (MBSs) is motivated in part by their non-Abelian characteristics and potential application in fault-tolerant quantum computations [10-20]. It was predicted that the signature of the MBSs may exhibit as a zero bias conductance peak in the normal metal/topological superconductor junctions, as has been observed in hybrid devices of superconductor and semiconductor nanowire [21-25] and in ferromagnetic iron atomic chains on the surface of superconducting lead [26]. Nevertheless, an unambiguous experimental verification remains elusive because zero-bias peaks can also have non-Majorana origins, such as the Kondo effect or disorder effect [27-31].

The signal of current noise cross correlation could provide an alternative, even confirmative proof, to verify the existence of MBSs. In non-Majorana devices, it has been demonstrated experimentally that Cooper pairs can split into spin-entangled electrons flowing in two spatially separated normal metals, resulting in a positive current-current correlation [32-35]. Although many theoretical studies have been devoted to investigate the property of the noise cross correlation induced by MBSs [36-45], the Majorana-modulated nonlocal transport signal has not been reported experimentally up to now. One of the possible reasons is that the energy splitting of MBSs, which is essential to induce the noise cross correlation, is

\footnotetext{
*Corresponding author: luhz@sustc.edu.cn
}

usually weak. The Majorana energy splitting is at most tens of $\mu \mathrm{eV}$ in a topological nanowire with a length of 1-2 $\mu \mathrm{m}$ [46], and it approaches zero near the critical Zeeman field between the trivial and topological phases $[7,46]$. Compared to the Majorana energy splitting, other energy scales, such as the intra- and interdot Coulomb interaction and superconducting pairing energy, are much stronger. For instance, a typical value of the interdot Coulomb interaction is of the order of $0.1 \mathrm{meV}[47,48]$, and the pairing energy of a Cooper pair is of the order of $0.1 \mathrm{eV}$. Therefore, it is highly desirable to produce robust current-current correlations by combining the nonlocality of Majorana fermions and other robust physical mechanisms.

Recently, quantum transport properties modulated by Coulomb interactions in Majorana devices have attracted much attention [49-54]. In an interacting transistor coupled to MBSs, it is found that the conductance shows Coulomb oscillations with universal halving of the finite temperature peak under strong blockade conditions [51]. Altland and Egger studied multiple helical nanowires in proximity to a common mesoscopic superconducting island with a finite charging energy [52]. The MBSs prepared in a superconducting island with Coulomb interactions are suggested to realize the topological Kondo effect [53,54]. Béri and Cooper studied the simplest case with three leads coupled to two pairs of Majorana fermions with a charging energy [53]. The superconducting Coulomb island supporting MBSs offers a new playground to generate nonlocal current-current correlations in the absence of a finite Majorana energy splitting. However, since the large global charging energy is itself a strong nonlocal perturbation, it becomes troublesome whether the measured nonlocal current correlations are essentially generated by the charging energy or MBSs. It has been pointed out that Poissonian shot noises are generically obtained by MBSs in a floating topological superconductor in the absence of charging energy [55]. However, it does not provide a 
unique signature to confirm the existence of MBSs. Although the Coulomb-modulated conductance properties in Majorana devices have been well studied, it remains unknown how Coulomb interaction affects the nonlocal current noise cross correlations in Majorana systems.

In this paper, we investigate the nonlocal transport modulated by the Coulomb interactions in devices comprising a Majorana nanowire contacted to quantum dots and leads, where the nanowire is in proximity to a mesoscopic superconducting island with a finite charging energy. The purpose to insert the quantum dots is to offer an efficient way to modulate the nonlocal current correlations without affecting the property of the MBSs. The paper is organized as follows. In Sec. II, we introduce the model Hamiltonian of the Majorana-dot device fabricated on a superconducting Coulomb island, as well as the current and noise cross correlation formulas. In Sec. III, we investigate the nonlocal transport properties modulated by the charging energy in the island. We consider the cases that the superconducting island is floating and connected to a bulk superconductor, respectively. For comparison, the current noise cross correlation property in the absence of MBSs is also discussed. Finally, a summary is given in Sec. IV.

\section{MODEL AND FORMALISM}

\section{A. Model Hamiltonian}

A schematic of the Coulomb-Majorana junction is illustrated in Fig. 1. We consider a semiconductor nanowire proximity coupled to a mesoscopic superconducting island with a finite charging energy, and there exists a pair of MBSs at the wire ends under a proper magnetic field [9]. Each end of the nanowire connects to a normal metal electrode via a quantum dot. The intraisland Coulomb interactions introduce correlations between the two MBSs and thereby generate

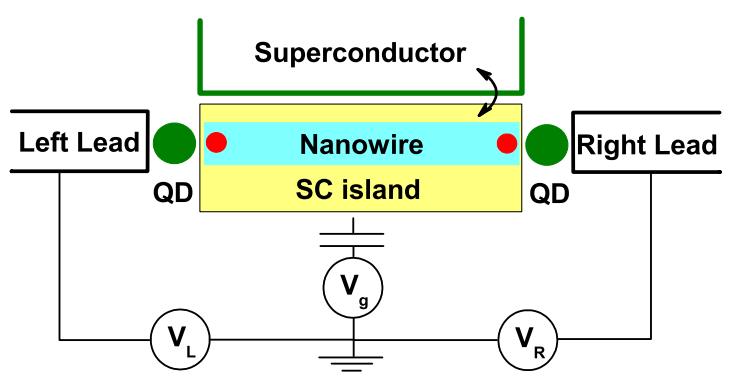

FIG. 1. Schematic view of the device. A Majorana nanowire is proximity coupled to a mesoscopic superconducting (SC) island with a finite charging energy. The nanowire is in the topological superconducting phase and a pair of MBSs (marked as the red dots) appear in the wire ends. Each end of the nanowire is connected to a normal metal electrode via a quantum dot (QD). In addition, the island is coupled to another grounded bulk superconductor. The dimensionless gate parameter $n_{g}$ (see main text) is proportional to a gate voltage $V_{g}$ that controls the average charge on the SC island. The leads are biased with the chemical potential $V_{L}$ and $V_{R}$. Crossed Andreev reflections can be induced by correlating the currents that flow into the topological nanowire via the MBSs. The nonlocal current cross correlations could be generated by Coulomb interactions in the absence of the Majorana energy splitting. the cross correlation between the currents flowing through the two quantum dots. It is convenient to define nonlocal auxiliary fermion operators $\eta_{a}=f+f^{\dagger}, \eta_{b}=i\left(f^{\dagger}-f\right)$ for the MBSs, with the number operator $\hat{n}_{f}=f^{\dagger} f$. In the regular fermionic representation, the instantaneous charged state of the superconducting island is described by $\left(N_{c}, n_{f}\right)$, where the integer $N_{c}$ represents the Cooper pair number in the island and $n_{f}$ is the eigenvalue of $\hat{n}_{f}$, respectively. The total Hamiltonian is given by

$$
H=H_{w}+H_{d}+H_{c}+H_{b}+H_{l}+H_{t} .
$$

The nanowire is in the topological superconducting state and isolated MBSs appear at the wire ends. Including the Majorana energy splitting and a Coulomb charging term, the island Hamiltonian is fully expressed by [51]

$$
H_{w}=\varepsilon_{M}\left(f^{\dagger} f-1 / 2\right)+E_{c}\left(2 \hat{N}_{c}+\hat{n}_{f}-n_{g}\right)^{2},
$$

where $\varepsilon_{M}$ denotes the Majorana energy splitting and the capacitive charging effect is denoted by $E_{c}$ and can be tuned by a gate voltage parameter $n_{g} \propto V_{g}$. Experimentally, the charging energy in a superconducting island can reach at about $100 \mu \mathrm{eV}$ [56,57]. Next, the two quantum dots in Fig. 1 are assumed to be in the Coulomb blockade regime such that each dot can be modeled as a single fermion level. The energy level in each dot can be tuned by gate voltages, and the spin degeneracy on the dots also breaks by the Zeeman field which induces the topological superconducting phase of the wire. As a consequence, we can use the effectively spinless fermion operators $d_{j}^{\dagger}\left(d_{j}\right)$ for the dots [58-60]. The Hamiltonian of the quantum dots reads

$$
H_{d}=\varepsilon_{1} d_{1}^{\dagger} d_{1}+\varepsilon_{2} d_{2}^{\dagger} d_{2},
$$

where $\varepsilon_{j}$ denotes the dot energy level and $d_{j}^{\dagger}\left(d_{j}\right)$ represents the electron creation (annihilation) operator on dot $j$.

Different from the noninteracting case in which $E_{c}=0$, there exists an energy cost when absorbing or emitting a Cooper pair in the superconducting island. To ensure the charge conservation, it is essential to add a factor $e^{\mp i \varphi}$ in the tunneling terms $d_{j}^{\dagger} f^{\dagger}\left(d_{j} f\right)$, which changes the Cooper pair number $N_{c}$ by one unit and thus restores the charge balance. The Hamiltonian of the Majorana-dot tunneling then reads [58-60]

$$
H_{c}=\lambda_{1} d_{1}^{\dagger}\left(e^{-i \varphi} f^{\dagger}+f\right)+\lambda_{2}\left(e^{-i \varphi} f^{\dagger}-f\right) d_{2}^{\dagger}+\text { H.c. },
$$

where $\lambda_{1,2}$ denotes the dot-Majorana coupling strength and the operator $e^{ \pm i \varphi}$ raises (lowers) the Cooper pair number by one unit, i.e., $N_{c} \rightarrow N_{c} \pm 1$. In conjunction with spin-orbit coupling and the Zeeman splitting, the nanowire in the topological phase resembles an effective $p$-wave superconductor. Therefore, the Cooper pair exchange with the bulk superconductor involves the spin-flip scattering processes. The spin properties of the topological nanowire as well as spin-flip processes in the contact can be fully taken into account via the tunnel couplings $\lambda_{1,2}$ and $E_{J}$, where $E_{J}$ denotes the Cooper pair exchange strength between the nanowire and the bulk superconductor. The tunnel couplings capture the possible spin dependence of microscopic transition amplitudes and can be 
taken as real-valued positive [58]. We include the Cooper pair exchange between the superconducting island and another bulk superconductor,

$$
H_{b}=-E_{J} \cos \varphi,
$$

with the Josephson coupling $E_{J}$.

Finally, the electrodes and the dot-electrode tunneling are described by the Hamiltonians

$$
\begin{aligned}
& H_{l}=\sum_{j k} \varepsilon_{j k} a_{j k}^{\dagger} a_{j k}, \\
& H_{t}=\sum_{j k}\left(t_{j} a_{j k}^{\dagger} d_{j}+\text { H.c. }\right),
\end{aligned}
$$

where $a_{j k}^{\dagger}\left(a_{j k}\right)$ is the electron creation (annihilation) operator in the lead $j$ with an energy $\varepsilon_{j k}$ and $t_{j}$ is the lead-dot coupling strength.

The current noise cross correlation has been well studied in a dot-Majorana-dot structure when the Majorana nanowire is directly in proximity with a bulk superconductor [40-45]. In this case, a finite Majorana energy splitting is essential to generate nonlocal correlations. In contrast, here the cross correlations could be controlled by the charging energy of the superconducting island as well as the Majorana energy splitting. If we consider a one-dimensional tight-binding Kitaev model [13], the two quantum dots look like two extended sites of the Kitaev model. In this sense, the Majorana zero modes will be located near the interface between the nanowire and the quantum dots, where the tail part of the wave function of the zero modes could enter the quantum dots. Differently, the dot energy levels could be tunable by applying gate voltages. If the dot energy levels are tuned to be high enough, the wave function of Majorana mode will appear hardly at the quantum dots.

\section{B. Diagonalized master equation approach}

We exploit the diagonalized master equation (DME) approach to investigate the electronic transport through this system in the sequential tunneling regime [61-66]. In our previous paper [45], the applicability of the DME approach has been discussed in the Majorana devices, by comparing with the nonequilibrium Green's function (NEGF) method. It is shown that the DME works well in most regimes of system parameters, while it breaks down for strong central region-lead coupling or when energy degeneracies appear. Here we extend our discussion to the interacting case. Compared to the NEGF method, the DME approach is convenient in dealing with many interacting energy levels, and there is strong coherence between different levels.

In the DME approach, we firstly diagonalize the Hamiltonian of the island-dot part and obtain the eigenvalues $E_{n}$ and their corresponding eigenfunctions $\left|\beta_{n}\right\rangle$. Different from the case that the superconductor is grounded directly, a superconducting island with a finite charging energy is used in the proposed tunneling device. To ensure the charge conservation, the total Cooper pair number $N_{c}$ should also be considered as a degree of freedom in the calculation [60]. To give an explicit matrix form of the island-dot Hamiltonian, it is convenient to index the states with the occupation numbers

$$
\left|n_{1} n_{2} n_{f} N_{c}\right\rangle=\left(d_{1}^{\dagger}\right)^{n_{1}}\left(d_{2}^{\dagger}\right)^{n_{2}}\left(f^{\dagger}\right)^{n_{f}}\left|000 N_{c}\right\rangle .
$$

Here the quantum numbers can take the values $n_{j}, n_{f}=0,1$ and $N_{c}=-N_{m}, \ldots N_{m}$, where $N_{m}$ is the cutoff for the Cooper pair number.

In the Born-Markov approximation, the time evolution of the density matrix $\rho_{D}(t)=\left\{\left|\beta_{n}\right\rangle\left\langle\beta_{n^{\prime}}\right|\right\}$ in terms of the states $\left|\beta_{n}\right\rangle$ is given by the rate equations

$$
\frac{d}{d t} \rho_{D}(t)=\mathbf{W} \rho_{D}(t)
$$

where the elements of the rate matrix are given by $[40,65]$

$$
\begin{aligned}
W_{n^{\prime} n}= & \sum_{j} \Gamma_{j}\left[f\left(\Delta_{n^{\prime} n}+\mu_{j}\right)\left|\left\langle\beta_{n^{\prime}}\left|d_{i}\right| \beta_{n}\right\rangle\right|^{2}\right. \\
& \left.+f\left(\Delta_{n^{\prime} n}-\mu_{j}\right)\left|\left\langle\beta_{n^{\prime}}\left|d_{j}^{\dagger}\right| \beta_{n}\right\rangle\right|^{2}\right]
\end{aligned}
$$

for $n \neq n^{\prime}$, and

$$
W_{n n}=-\sum_{n^{\prime} \neq n}^{N} W_{n^{\prime} n} .
$$

Here $f_{j}(\omega)=\left[1+e^{\omega / k_{B} T}\right]^{-1}$ is the Fermi-Dirac distribution function, $\mu_{j}$ is the chemical potential in lead $j$, and $\Delta_{k^{\prime} k}$ is the Bohr frequency of the transition from $\left|\beta_{k}\right\rangle$ to $\left|\beta_{k^{\prime}}\right\rangle$. In the wide-band limit approximation, the dot-lead coupling for dot $j$ is measured by the parameter $\Gamma_{j}=2 \pi\left|t_{j}\right|^{2} \rho_{j}$, with $\rho_{j}$ the spinless density of states near the Fermi surface of lead $j$.

The steady-state current $I_{j}$ is given by

$$
I_{j}=\sum_{k}\left[\hat{\Gamma}^{j} \rho_{D}^{(0)}\right]_{k},
$$

where $\rho_{D}^{(0)}$ is the steady-state solution of Eq. (8), $\hat{\Gamma}^{j}$ is the matrix of the current operator, and its elements are given by

$$
\begin{aligned}
\hat{\Gamma}_{k^{\prime} k}^{j}= & \Gamma_{j}\left[f\left(\Delta_{k^{\prime} k}+\mu_{j}\right)\left|\left\langle\beta_{k^{\prime}}\left|d_{j}\right| \beta_{k}\right\rangle\right|^{2}\right. \\
& \left.-f\left(\Delta_{k^{\prime} k}-\mu_{j}\right)\left|\left\langle\beta_{k^{\prime}}\left|d_{j}^{\dagger}\right| \beta_{k}\right\rangle\right|^{2}\right] .
\end{aligned}
$$

The first and the second terms of $\hat{\Gamma}_{k^{\prime} k}^{j}$ represent the tunneling amplitudes flowing into and out of the lead, respectively.

We are focusing on the current noise correlations modulated by the charging energy on the superconducting island. It is well known that the noise power spectra can be expressed as the Fourier transform of the current-current correlation function

$$
S_{I_{i} I_{j}}(\omega)=2\left\langle I_{i}(t) I_{j}(0)\right\rangle_{\omega}-2\left\langle I_{i}\right\rangle_{\omega}\left\langle I_{j}\right\rangle_{\omega} .
$$

Here, $I_{i}$ and $I_{j}$ are the electrical currents across dot $i$ and dot $j$, respectively, and $t$ is the time. Furthermore, the current-current correlation function of the currents $I_{i}$ and $I_{j}$ can be expressed in terms of the density matrix as

$$
\begin{aligned}
\left\langle I_{i}(t) I_{j}(0)\right\rangle= & \theta(t) \sum_{k}\left[\hat{\Gamma}^{i} \hat{T}(t) \hat{\Gamma}^{j} \rho^{(0)}\right]_{k} \\
& +\theta(-t) \sum_{k}\left[\hat{\Gamma}^{j} \hat{T}(-t) \hat{\Gamma}^{j} \boldsymbol{\rho}^{(0)}\right]_{k},
\end{aligned}
$$

with $\hat{T}(t)=\exp [\mathbf{W} t]$ the propagator governing the time evolution of the density matrix element $\rho_{k}(t)$. Finally, the 
current-current correlation in the $\omega$ space becomes

$$
\left\langle I_{i}(t) I_{j}(0)\right\rangle_{\omega}=\sum_{k}\left[\hat{\Gamma}^{i} \hat{T}(\omega) \hat{\Gamma}^{j} \boldsymbol{\rho}^{(0)}+\hat{\Gamma}^{j} \hat{T}(-\omega) \hat{\Gamma}^{i} \boldsymbol{\rho}^{(0)}\right]_{k},
$$

where $\hat{T}( \pm \omega)=(\mp i \omega \hat{I}-\mathbf{W})^{-1}$.

\section{NUMERICAL RESULTS AND DISCUSSION}

In the following, we present numerical results for the sequential tunneling currents and their correlations in the presence of the charging energy on the superconducting island in the nonlinear response regime. The negative noise cross correlation is related to the antibunching between tunneling events, which arises as a result of the Pauli exclusive principle of scattered electrons, while the positive cross correlation is usually related to the bunching of tunneling processes, e.g., due to the interchannel Coulomb blockade or crossed Andreev reflection [32-35]. In the calculation, we adopt the symmetric coupling strength as $\Gamma_{1,2}=\Gamma_{0}$ and $\lambda_{1,2}=10 \Gamma_{0}$, where $\Gamma_{0}$ serves as a convenient energy unit. The energy levels $\varepsilon_{1,2}$ of the quantum dots are tunable by applying gate voltages.

For comparison, we separately discuss two different cases: one is with $E_{J}=0$ and the other is with $E_{J} \gg \Gamma_{0}, \lambda_{0}, k_{B} T$. The case of $E_{J}=0$ corresponds to a floating superconducting island, and the electrons in the island can only tunnel through the quantum dots. For $E_{J}=0$, the current flows from the left lead to the right lead when a bias voltage $V_{b}$ is applied and we take $\mu_{L}=-\mu_{R}=V_{b} / 2$ in the calculation. For $E_{J} \gg$ $\Gamma_{0}, \lambda_{0}, k_{B} T$, the island is strongly coupled to the bulk superconductor. In this case, we take $\mu_{L}=\mu_{R}=V_{0}$ and the currents flow from the two electrodes into the superconducting island. The sign of the current noise cross correlation sensitively depends on the interplay between the intraisland Coulomb interaction and the crossed Andreev reflection. In the limit that $E_{C} \rightarrow 0$ and $E_{J} \rightarrow \infty$, the result should reduce to the case when the bulk superconductor is grounded, which has been well studied previously [40-45].

\section{A. Floating superconducting island $\left(E_{J}=0\right)$}

Firstly we address the $n_{g}$ dependence of the linear conductance $\left(V_{b} \rightarrow 0\right)$, as shown in Fig. 2(a) for the symmetric dot energy level configuration $\left(\varepsilon_{1}=\varepsilon_{2}\right)$, and in Fig. 2(b) for the antisymmetric level configuration $\left(\varepsilon_{1}=-\varepsilon_{2}\right)$. In both configurations, the conductance shows clear $n_{g}$ dependent oscillations in addition to a constant part. With the increase of the charging energy $E_{c}$, the constant part is considerably suppressed while the oscillation amplitude increases. As shown in the charging part of $H_{w}$ in Eq. (2), a shift $n_{g} \rightarrow$ $n_{g} \pm 2$ can be compensated by absorbing or emitting a Cooper pair $N_{c} \rightarrow N_{c} \pm 1$. Therefore, all observables are periodic in $n_{g}$ with a period of $\Delta n_{g}=2$. This feature is different from the case when the MBSs are directly coupled to the two electrodes, in which a zero-energy Majorana mode $\left(\varepsilon_{M}=0\right)$ is considered and the oscillation period is $\Delta n_{g}=1$ [51]. When Majorana fermions are directly coupled to two leads, the parity change in the superconducting island costs a finite energy and the period becomes $\Delta n_{g}=2$ for $\varepsilon_{M} \neq 0$. When two quantum dots are inserted, the period is $\Delta n_{g}=2$ for either $\varepsilon_{M}=0$ or

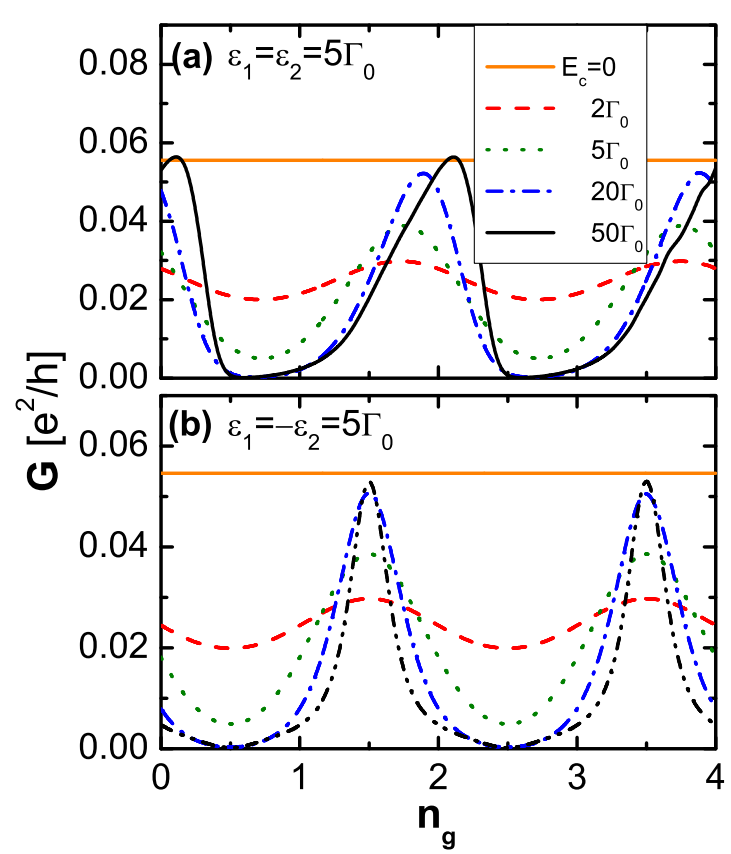

FIG. 2. The linear conductance $G$ as a function of $n_{g}$ for different charging energies $E_{c}$ on the superconducting island. (a) The symmetric configuration of dot energy levels $\left(\varepsilon_{1}=\varepsilon_{2}\right)$. (b) The antisymmetric dot level configuration $\left(\varepsilon_{1}=-\varepsilon_{2}\right)$. Other parameters: $E_{J}=0, k_{B} T=2 \Gamma_{0}, \varepsilon_{1}=5 \Gamma_{0}, \varepsilon_{M}=0, \lambda_{1,2}=10 \Gamma_{0}$, and $\Gamma_{0}$ is taken as the energy unit.

$\varepsilon_{M}>0$. In Fig. 2, it is indicated that the value of conductance is much smaller than $e^{2} / h$. This is because a relatively high temperature $\left(k_{B} T=2 \Gamma_{0}\right)$ is adopted in the calculation. In this case, the conductance would be considerably suppressed compared to the case of zero temperature [51].

Another feature in Fig. 2 is that the conductance curves are symmetric about $n_{g}=1 / 2$ for the antisymmetric dot level configuration, which is absent for the symmetric case $\varepsilon_{1}=\varepsilon_{2}$. By applying a particle-hole transformation that exchanges the creation and annihilation operators, the total Hamiltonian is invariant under the replacement $n_{g} \rightarrow 1-n_{g}$ for $\varepsilon_{1}=-\varepsilon_{2}$. Due to the periodicity, the observables are symmetric about $n_{g}=2 n+1 / 2$, where $n$ is an integer.

The effects of the charging energy on the transport properties are presented in Fig. 3. Due to the current conservation $I_{1}=-I_{2}$, the current correlations in a floating two-terminal setup obey the relationship $S_{11}=S_{22}=-S_{21}=-S_{12}=S$. Compared to the noninteracting case, the most important difference in the transport is that the Coulomb interaction could induce a negative differential conductance (NDC) and strongly enhance the shot noise. As the bias voltage increases from $V_{b}=0$, the current appears and increases gradually when the voltage sweeps through the lowest positive eigenenergy of the central region. However, as the lead voltage increases and other energy levels lie in the transport window, the charging energy induces a strong competition between different tunneling paths, leading to a decrease of the current. In Fig. 3(a), the current shows an oscillating behavior for small charging energies. As the bias voltage $V_{b}$ increases, the Cooper pair states $\left|N_{c}\right\rangle$ are involved in the transport one by one, resulting in the current oscillation. A similar behavior can also be observed 


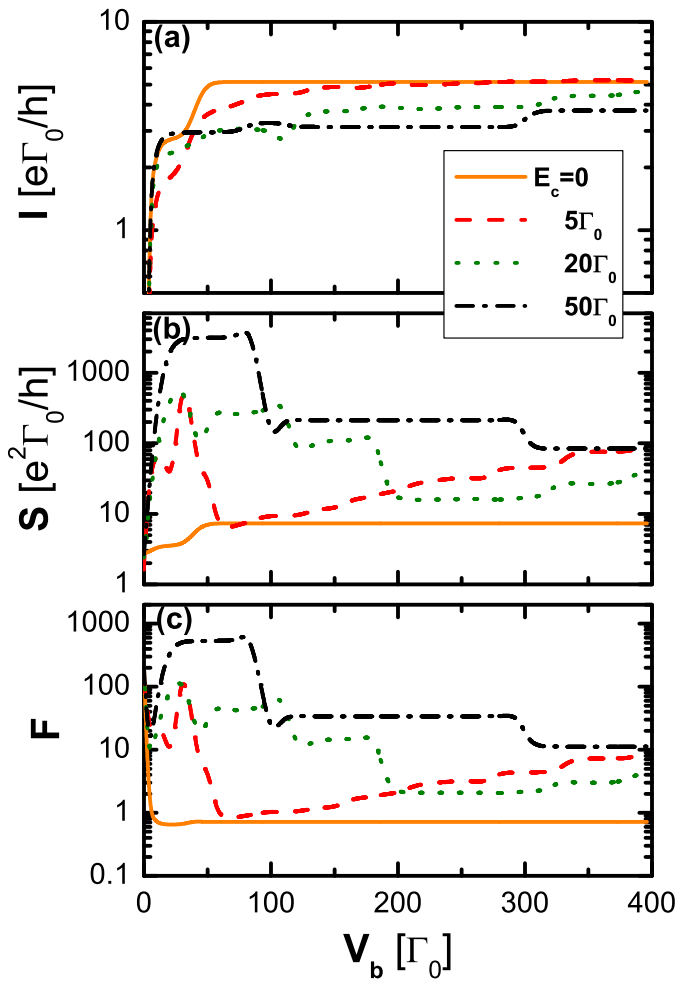

FIG. 3. (a) The current $I$, (b) the shot noise $S$, and (c) the Fano factor $F$ as functions of the bias voltage $V_{b}$ for different charging energies $E_{c}$. Other parameters: $E_{J}=0, k_{B} T=2 \Gamma_{0}, \varepsilon_{1}=\varepsilon_{2}=5 \Gamma_{0}$, $\varepsilon_{M}=0, \lambda_{1,2}=10 \Gamma_{0}$, and $n_{g}=0$.

in the bias voltage dependence of the shot noise, as shown in Fig. 3(b). A giant shot noise $S$ can be generated in the Coulomb blockade regime. One usually introduces the Fano factor $F=S / 2 e I$ to represent the deviation from Poissonian shot noise for which $F=1$. The noise Fano factor $F$ is demonstrated as a function of the bias voltage in Fig. 3(c). In the noninteracting case $E_{c}=0$, the shot noise is always a sub-Poissonian type, i.e., $F<1$. In the presence of a finite charging energy, the super-Poissonian shot noise $(F>1)$ is induced in most transport regimes.

The NDC and the giant shot noise $S$ arise from the same mechanism, known as the dynamical channel blockade effect. Different from the Schottky noise, which is independent of the frequency, the shot noise reflects the dynamical tunneling correlations and is frequency dependent. In the case of a floating superconducting island $\left(E_{J}=0\right)$, the electrons in the island can only tunnel through the quantum dots. In this case, the superconducting island behaves like a normal quantum dot with multiple interacting energy levels. The giant shot noise has been demonstrated in an interacting quantum dot with multiple tunneling channels in several previous studies [67-74]. The reason is traced to a dynamical channel blockade of the mechanically aided shuttle current that occurs in devices with high polarization or asymmetry of the channel-lead coupling strengths. In Fig. 4 we present the dependence of state populations in the superconducting island on the charging energy $E_{c}$ to demonstrate the coupling asymmetry. By tracing out the freedoms of $n_{1,2}$ in two quantum dots, one can obtain the state distribution $\rho\left(n_{f}, N_{c}\right)$ in the island. For $E_{c}=0$, all

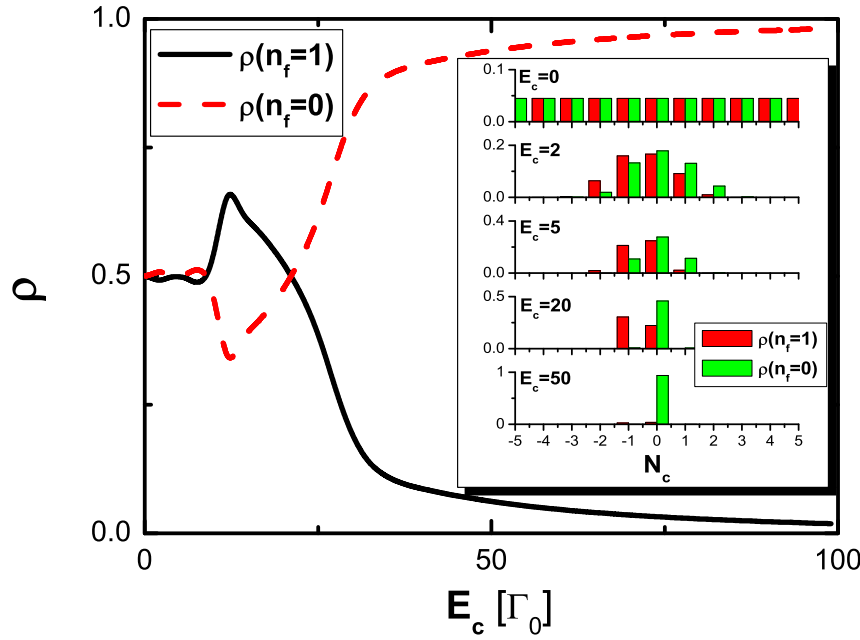

FIG. 4. The state population $\rho\left(n_{f}\right)$ in the island as a function of the charging energy. The inset shows the distribution of $\rho\left(n_{f}\right)$ as a function of the Cooper pair number $N_{c}$. For $E_{c}=0$, all the states $\left|n_{f}, N_{c}\right\rangle$ in the island have equal probability and we take a cutoff of the Cooper pair number $\left(N_{c} \in[-5,5]\right)$. Other parameters: $V_{b}=$ $30 \Gamma_{0}, E_{J}=0, k_{B} T=2 \Gamma_{0}, \varepsilon_{1}=\varepsilon_{2}=5 \Gamma_{0}, \varepsilon_{M}=0, \lambda_{1,2}=10 \Gamma_{0}$, and $n_{g}=0$.

the states $\left|n_{f}, N_{c}\right\rangle$ in the island have equal probability and $\rho\left(n_{f}=1\right)=\rho\left(n_{f}=0\right)=1 / 2$. In the presence of the charging energy, the eigenenergies of the Majorana-dot device become $E_{c}$ dependent and the degeneracy is lifted. This dependence remarkably modifies the electronic occupations in comparison with the noninteracting case. For the strong charging energy, the island prefers to occupy the state $\mid n_{f}=$ $\left.0, N_{c}=0\right\rangle$ and the occupation probabilities of other states are strongly suppressed. With the increase of bias voltage, more eigenenergy levels of Majorana-dot part enter the transport window. The long time occupation of the state $\mid n_{f}=0, N_{c}=$ $0\rangle$ impedes the entry of electrons into the island region through other channels, leading the suppression of the current and enhancement of shot noise. This mechanism has been illustrated in several quantum transport systems, such as multilevel quantum dot devices [70-74], Franck-Condon blockade in single molecules [75], and nanoscale oscillators [76].

The picture presented above is based on the sequential tunneling regime. In the Coulomb blockade regime, the first-order tunneling is exponentially suppressed, while higher-order processes can contribute a small current. For a weak dot-lead coupling strength and high temperature, the current contributed by higher-order tunneling processes is rather small, which cannot remove the interchannel blockade remarkably. To elucidate the role of higher-order tunneling processes, a relatively high temperature $\left(k_{B} T=2 \Gamma_{0}\right)$ has been considered in our calculation, in which the current induced by thermal fluctuations is activated. In this case, the current contributed by higher-order tunneling processes is overwhelmed by thermal currents.

\section{B. Strong coupling between superconducting island and a bulk superconductor $\left(E_{J} \gg \Gamma_{0}\right)$}

Now we consider a three-terminal case in which the superconducting island is strongly coupled to the bulk 
superconductor. In this case, the Cooper pairs can tunnel freely between the island and the bulk superconductor. All states $\left|N_{c}\right\rangle$ are strongly mixed. To investigate the current noise cross correlation modulated by the charging energy, symmetric chemical potentials $\mu_{1}=\mu_{2}=V_{0}$ are applied to the two electrodes, and the currents flow from the two electrodes to the superconducting island.

In the noninteracting case where $E_{c}=0$, the degree of freedom of Cooper pair number $N_{c}$ decouples from those of the fermionic part $\left|n_{1} n_{2} n_{f}\right\rangle$ in the limit $E_{J} \rightarrow \infty$. Correspondingly, the transport properties reduce to the results for a noninteracting dot-MBS-dot structure, which has been well investigated previously [40-45]. To verify this physical picture, we present the current noise cross correlation $S_{12}$ for different $E_{J}$, as demonstrated in Fig. 5. For comparison, we also show the results when the semiconductor nanowire is in proximity to a grounded superconductor. We take $E_{c}=0$ and $\varepsilon_{M}=10 \Gamma_{0}$ to ensure that the noise cross correlation is only induced by the Majorana energy splitting. For the grounded case, the cross correlation $S_{12}$ is exactly solved by using the NEGF method [45]. According to Fig. 5, even for $E_{J}=50 \varepsilon_{M}$, $S_{12}$ shows quite different behavior, compared to that in the directly grounded case. However, when $E_{J}$ is of the order of $100 \varepsilon_{M}$, the cross correlations in the two cases are in good agreement with each other in all regimes of the lead chemical potentials. Above this strength of $E_{J}$, the superconducting island can be regarded as being grounded directly.

The discussion above serves as a ground to extend the discussion to the situation in which the current cross correlation is purely induced by the charging energy in the superconducting island. The Hamiltonian of the central region is exactly diagonalized in the DME approach. The approximation of the DME approach is that the dot-lead coupling strength is taken

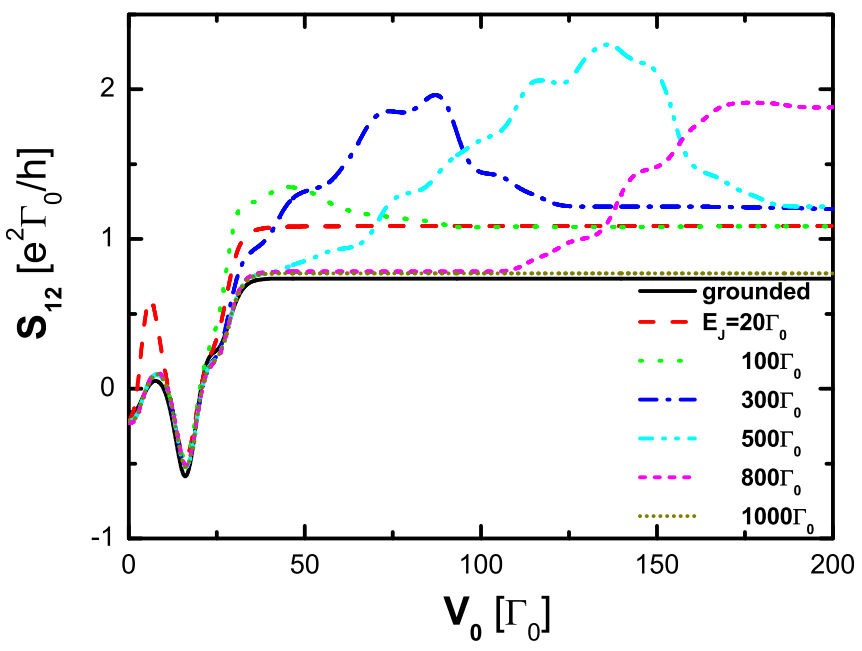

FIG. 5. The zero-frequency current noise cross correlation $S_{12}$ as a function of the lead voltage $V_{0}$ for different $E_{J}$ at $E_{c}=$ 0 . The cross correlation is induced by a finite Majorana energy splitting $\varepsilon_{M}=10 \Gamma_{0}$. The black line corresponds to the case in which the semiconductor nanowire is in proximity to the grounded superconductor. The result denoted by the black line is exact and obtained by using the nonequilibrium Green's function method. Other parameters: $k_{B} T=2 \Gamma_{0}, \varepsilon_{1}=\varepsilon_{2}=5 \Gamma_{0}, \lambda_{1,2}=10 \Gamma_{0}$, and $n_{g}=0$.
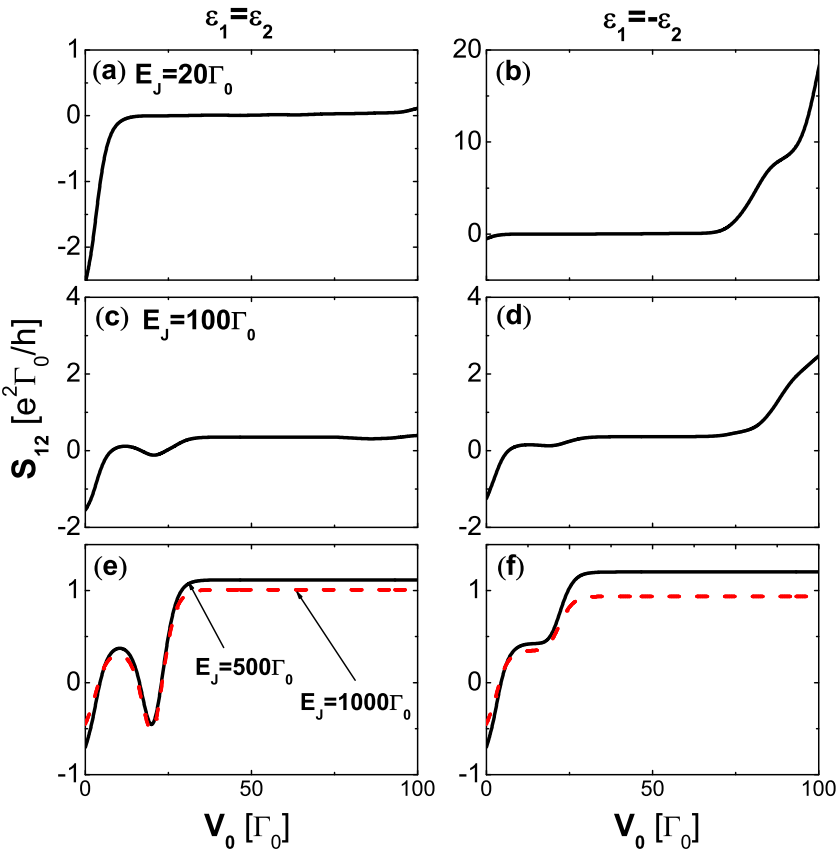

FIG. 6. The zero-frequency current noise cross correlation $S_{12}$ as a function of the lead chemical potentials $\mu_{1}=\mu_{2}=V_{0}$ for different $E_{J}$ at $\varepsilon_{M}=0$. We consider the symmetric configuration of the quantum dot levels in (a) $E_{J}=20 \Gamma_{0}$, (c) $E_{J}=100 \Gamma_{0}$, (e) $E_{J}=500 \Gamma_{0}, 1000 \Gamma_{0}$ and the antisymmetric level configuration in (b) $E_{J}=20 \Gamma_{0}$, (d) $E_{J}=100 \Gamma_{0}$, (f) $E_{J}=500 \Gamma_{0}, 1000 \Gamma_{0}$, respectively. The cross correlation is induced by the charging energy $E_{c}=30 \Gamma_{0}$. For strong couplings with the bulk superconductor, such as $E_{J}=500 \Gamma_{0}$ or $1000 \Gamma_{0}$, the Coulomb blockade induced by the charging energy is smeared out. This case is equivalent to the case that the superconducting island is grounded directly. Other parameters: $k_{B} T=2 \Gamma_{0}, \varepsilon_{1}=5 \Gamma_{0}, \lambda_{1,2}=10 \Gamma_{0}$, and $n_{g}=0$.

as a perturbation parameter and is much weaker than other system energy scales.

In Fig. 6, we demonstrate the current noise cross correlation $S_{12}$ as a function of the lead chemical potentials $\mu_{1}=\mu_{2}=V_{0}$. To ensure that the cross correlation is induced only by the charging energy, we take $\varepsilon_{M}=0$ and $E_{c}=30 \Gamma_{0}$. We consider the symmetric $\left(\varepsilon_{1}=\varepsilon_{2}\right)$ and antisymmetric $\left(\varepsilon_{1}=-\varepsilon_{2}\right)$ configurations of the dot levels. For the cross correlation induced by the Majorana energy splitting, $S_{12}$ is always positive in the antisymmetric level configuration [40,41]. However, Fig. 6 shows that the current cross correlation $S_{12}$ modulated by the charging energy is always negative in both level configurations at small lead chemical potentials. For small $V_{0}$, the device lies in the Coulomb blockade regime. The transport through the two dots compete with each other, leading to a negative $S_{12}$. With the increase of $V_{0}$, more eigenenergy levels of the dot-Majorana island-dot part are involved in the transport and the Coulomb blockade is partly relieved. The crossed Andreev reflection becomes dominant gradually, resulting in a positive cross correlation. The coupling $E_{J}$ between the island and the bulk superconductor helps to remove the dynamical Coulomb blockade. For a strong $E_{J}$, the Coulomb blockade induced by the charging energy is smeared out. This case is equivalent to the case in which the superconducting island is 


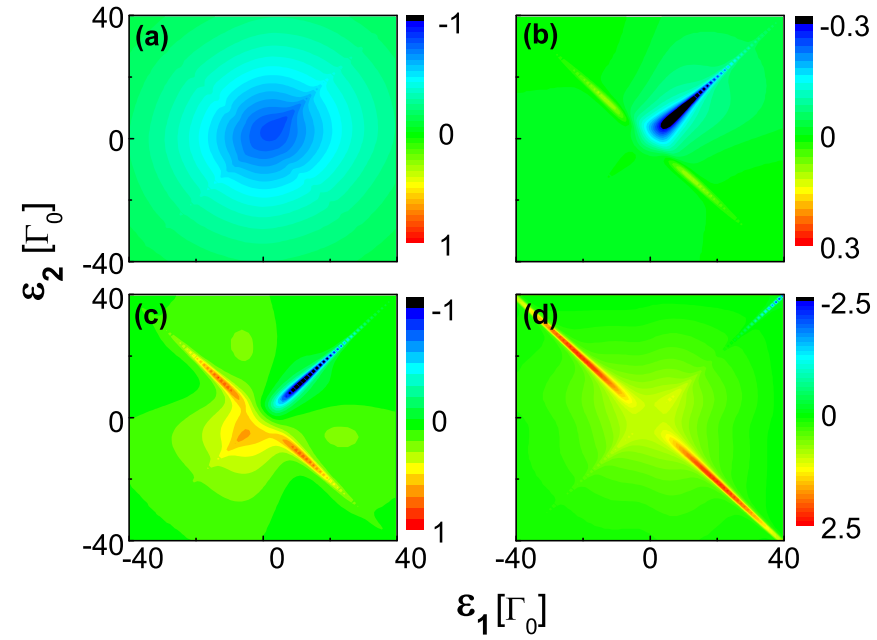

FIG. 7. The zero-frequency current noise cross correlation $S_{12}$ as a function of the dot energy levels $\varepsilon_{1}$ and $\varepsilon_{2}$ for different lead chemical potentials $\mu_{1}=\mu_{2}=V_{0}$. (a) $V_{0}=2 \Gamma_{0}$, (b) $10 \Gamma_{0}$, (c) $20 \Gamma_{0}$, and (d) $50 \Gamma_{0}$. A strong coupling $E_{J}=500 \Gamma_{0}$ between the island and the bulk superconductor is considered. Other parameters: $E_{c}=30 \Gamma_{0}$, $\varepsilon_{M}=0, k_{B} T=5 \Gamma_{0}, \lambda_{1,2}=10 \Gamma_{0}$, and $n_{g}=0$.

grounded directly. For a small $E_{J}=20 \Gamma_{0}$, Fig. 6(b) shows that the current cross correlation is strongly enhanced, due to the nonlocality of the MBSs and the dynamical channel blockade effect. Such an enhancement of the current cross correlation provides a new signature for the existence of the MBSs.

The quantum dot offers an approach to control and modulate the MBSs and related nonlocal transport by tuning the dot energy levels. Figures 7 and 8 demonstrate the current noise cross correlation $S_{12}$ as a function of the dot energy levels $\varepsilon_{1}$ and $\varepsilon_{2}$ for different chemical potentials $\mu_{1}=\mu_{2}=V_{0}$ in the strong and weak $E_{J}$ limits, respectively. It is shown

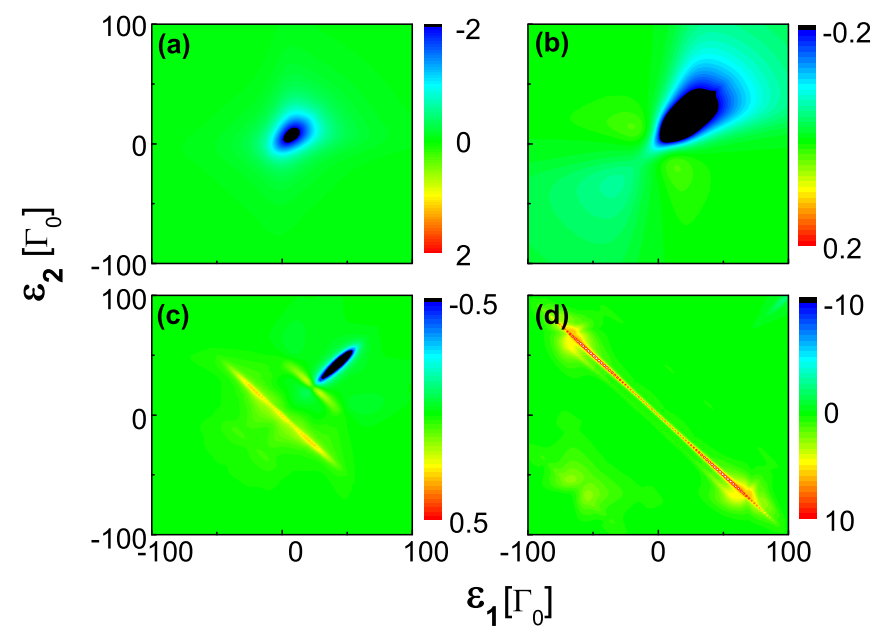

FIG. 8. The zero-frequency current noise cross correlation $S_{12}$ as a function of the dot energy levels $\varepsilon_{1}$ and $\varepsilon_{2}$ for different lead chemical potentials $\mu_{1}=\mu_{2}=V_{0}$. (a) $V_{0}=2 \Gamma_{0}$, (b) $20 \Gamma_{0}$, (c) $50 \Gamma_{0}$, and (d) $100 \Gamma_{0}$. The coupling between the island and the bulk superconductor is assumed to be comparable to the charging energy by taking $E_{J}=50 \Gamma_{0}$ and $E_{c}=30 \Gamma_{0}$. Other parameters: $\varepsilon_{M}=0, k_{B} T=5 \Gamma_{0}$, $\lambda_{1,2}=10 \Gamma_{0}$, and $n_{g}=0$. that for different $V_{0}$, the cross correlation shows distinct correlation patterns. For a small $V_{0}$, the currents are mainly contributed by the thermal activation, and only a negative cross correlation is induced and centered near $\varepsilon_{i}=0$. This feature is different from the correlation property induced by the Majorana energy splitting, in which a four-peak cloverlike pattern of noise cross correlation appears when tuning the dot energy levels $[40,41,45]$. With the increase of $V_{0}$, the cross correlation $S_{12}$ becomes positive gradually along the line of $\varepsilon_{1}=-\varepsilon_{2}$. The antisymmetric level configuration tends to enhance the crossed Andreev reflection processes. As $V_{0}$ increases further, $S_{12}$ in the region of $\varepsilon_{1,2}<0$ becomes positive. The sign reversal of the cross correlation occurs in the region of $\varepsilon_{1,2}>0$ when $V_{0}$ is large enough. In this regime, the transport channels through all eigenenergy levels are open and the competition between different tunneling paths leads to the positive $S_{12}$ in all regions. In this case, the cross correlation $S_{12}$ in the region of antisymmetric level configuration $\left(\varepsilon_{1}=-\varepsilon_{2}\right)$ is much stronger than those in other regions. When the coupling strength $E_{J}$ is comparable to the charging energy $E_{c}$, the enhanced current cross correlations appear in the large $V_{0}$ limit, as a result of the dynamical Coulomb blockade effect. When $E_{J}$ is strong enough, all the Cooper pair number states $\left|N_{c}\right\rangle$ are well mixed, which suppresses the Coulomb blockade effect. Therefore, the positive cross correlation in the strong $E_{J}$ limit becomes much weaker.

Above we consider the case of exact Majorana zero mode $\left(\varepsilon_{M}=0\right)$ to ensure that the current cross correlation is purely induced by the charging energy in the superconducting island. Now we turn to investigate the effect of a finite Majorana energy splitting. In the noninteracting case and at small voltages, previous studies [40-42,45] have shown that a finite Majorana energy splitting induces a four-peak cloverlike pattern of cross correlation with two positive parts (for $\varepsilon_{1} \varepsilon_{2}<0$ ) and two negative parts (for $\varepsilon_{1} \varepsilon_{2}>0$ ) when tuning the two dot energy levels. Figure 8 shows the current noise cross correlation $S_{12}$ as a function of dot energy levels for the case of symmetric $\left(\varepsilon_{1}=\varepsilon_{2}\right)$ and antisymmetric $\left(\varepsilon_{1}=\varepsilon_{2}\right)$ level configurations of two dots at small lead voltages. We take $\varepsilon_{M}=20 \Gamma_{0}$ and different intraisland charging energies. It is illustrated in Fig. 9 that a finite energy splitting $\varepsilon_{M}$ favors to suppress the negative cross correlation. In the noninteracting case $E_{c}=0$, the cross correlation is negative (positive) for the symmetric (antisymmetric) level configuration, consistent with the previous studies $[40-42,45]$. If $E_{c}$ is comparable to $\varepsilon_{M}$, the positive cross correlation is suppressed almost completely, while the negative cross correlation is enhanced. It is shown in Fig. 9 that a small charging energy $E_{c}$ could smear the four-peak cloverlike correlation pattern formed in the noninteracting case.

\section{Comparison with a non-Majorana setup}

For comparison, we also consider a non-Majorana setup. It consists of a superconductor island coupled to two quantum dots, and each dot is connected to a normal metallic electrode. In the case that the pairing strength in the superconducting island is much stronger than other system parameters, the quasiparticles in the superconductor are inaccessible, and one can trace out of the degrees of freedom of the superconducting 


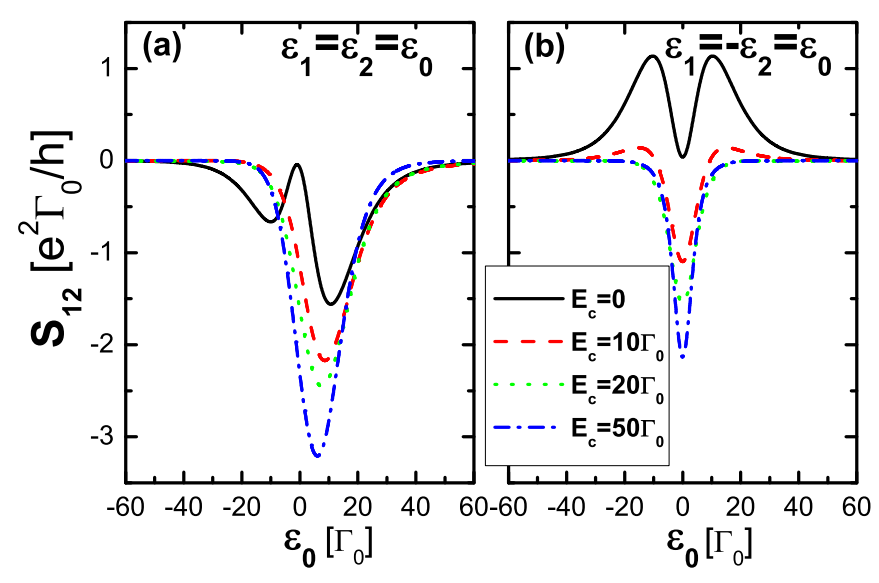

FIG. 9. The zero-frequency current noise cross correlation $S_{12}$ at (a) symmetric $\left(\varepsilon_{1}=\varepsilon_{2}\right)$ and (b) antisymmetric $\left(\varepsilon_{1}=-\varepsilon_{2}\right)$ level configurations of two dots for different intraisland charging energies $E_{c}=0,10 \Gamma_{0}, 20 \Gamma_{0}$, and $50 \Gamma_{0}$. A finite Majorana energy splitting $\varepsilon_{M}=20 \Gamma_{0}$ is considered. Other parameters: $E_{J}=100 \Gamma_{0}, V_{0}=2 \Gamma_{0}$, $k_{B} T=5 \Gamma_{0}, \lambda_{1,2}=10 \Gamma_{0}$, and $n_{g}=0$.

island without inducing any dissipative dynamics. An effective Hamiltonian to describe the dynamics of the double dots could be obtained by performing a real-time perturbative expansion [77,78]. In the limit of an infinite intradot Coulomb interaction, the effective model Hamiltonian of the dotssuperconducting island part is given by

$$
\begin{aligned}
H_{\mathrm{eff}}= & \varepsilon_{1} d_{1}^{\dagger} d_{1}+\varepsilon_{2} d_{2}^{\dagger} d_{2}+\Delta_{\mathrm{eff}}\left(d_{1}^{\dagger} d_{2}^{\dagger} e^{-i \varphi}+d_{2} d_{1} e^{i \varphi}\right) \\
& +E_{c}\left(\hat{N}_{c}-n_{g}\right)^{2}-E_{J} \cos \varphi
\end{aligned}
$$

Here $\Delta_{\text {eff }}$ is the effective pairing strength between the quantum dots, and it is determined by the coupling strength between the superconducting island and quantum dots. The pairing terms in $H_{\text {eff }}$ describe the formation of nonlocal superconducting correlations between the two dots induced by the splitting of Cooper pairs into the two dots.

Here we neglect the processes that electrons tunnel via the superconductor from one dot to the other, which involves virtual occupation of quasiparticle states above the gap. It should be noted that this device can still host MBS on each dot if the electron tunneling processes between two dots are considered [79]. Meanwhile, an inhomogeneous magnetic field is considered to make two dots fully spin-polarized, but in different directions with an angle $\theta$. The amplitude for tunneling between the dots therefore depends on the angle $\theta$ as $t=t_{0} \cos (\theta / 2)$, where $t_{0}$ is the tunneling amplitude for parallel fields. Similarly, the effective pairing $\Delta_{\text {eff }}$ also depends on the angle and is given by $\Delta_{\text {eff }}=\Delta_{0} \sin (\theta / 2)$, where $\Delta_{0}$ is the pairing strength between two dots for antiparallel spin polarizations. In the case of $t=\Delta_{\text {eff }}$ and either $\varepsilon_{1}$ or $\varepsilon_{2}$ being equal to zero, zero energy solutions of MBSs could be obtained [79]. When the superconducting gap is much larger than other energy scales, electrons tunneling between two dots via the superconductor will be strongly suppressed. Therefore, the validation of the non-Majorana setup we considered is that the processes of electrons tunneling between two dots via the superconductor are rather weak, i.e., $t \ll \Delta_{\text {eff }}$.

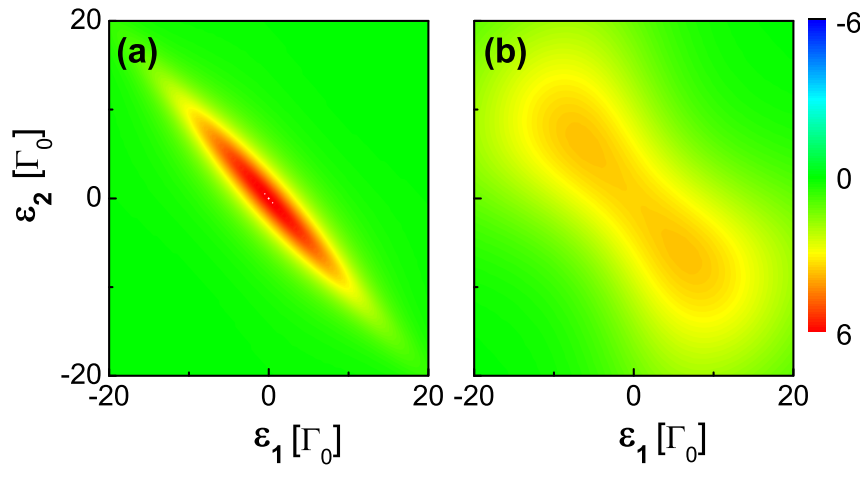

FIG. 10. The zero-frequency current noise cross correlation $S_{12}$ as functions of dot energy level $\varepsilon_{1}$ and $\varepsilon_{2}$ for (a) $E_{J}=20 \Gamma_{0}$ and (b) $E_{J}=500 \Gamma_{0}$ in the absence of the MBSs. Other parameters: $E_{c}=$ $30 \Gamma_{0}, V_{0}=2 \Gamma_{0}, k_{B} T=5 \Gamma_{0}, \lambda_{1,2}=10 \Gamma_{0}, n_{g}=0$, and $\Delta_{\text {eff }}=10 \Gamma_{0}$.

In the absence of MBSs, there are no other low-energy quasiparticle states in the superconducting island and electrons tunnel from the quantum dots into the island in pairs. In the limit of infinitely strong Coulomb interaction in the quantum dots, the currents are dominated by the crossed Andreev reflection. Only a positive current cross correlation could be generated, and the sign of the cross correlation is independent of the dot energy levels and the charging energy. In Fig. 10 the current noise cross correlation is plotted as a function of dot energy levels in the absence of MBSs. Only a positive cross correlation exists for the non-Majorana device. This is quite different from the results in the presence of MBSs. In Figs. 6-8, only a negative cross correlation is generated at low lead chemical potentials. Therefore, we can check the existence of MBSs from the sign of the current noise cross correlation at the small lead chemical potentials.

We have assumed that the on-site Coulomb interaction in the two dots is so strong that only one electron is allowed to stay in the dot at one time. In this case, the direct Andreev reflection processes are suppressed. Finally we briefly discuss the effect of the direct Andreev reflection processes on the current noise cross correlation. If the two dots are connected to a bulk superconductor, the crossed Andreev reflection induces positive cross correlation, while the direct Andreev reflection processes do not contribute to the cross correlation. However, if the two dots are coupled to a superconducting island with a finite charging energy, the direct Andreev reflection processes through one dot will compete with the tunneling in another dot to lower the energy in the island. In this case, it is expected that a negative cross correlation is induced.

\section{CONCLUSION}

In conclusion, we investigate the currents and their noise cross correlations modulated by a global charging energy in a hybrid device of MBSs and quantum dots. The MBSs are created in a semiconductor nanowire in proximity to a mesoscopic superconducting island. Each end of the nanowire is weakly connected to a quantum dot and a normal metal lead. This configuration is motivated by the possibility to switch the sign of current noise cross correlation by varying the dot energy levels. We have studied two cases: the superconducting 
island is floating, and the island is connected to another bulk superconductor. The floating island case shows a negative differential conductance and a giant super-Poissonian shot noise, resulting from the interplay of the nonlocality of the MBSs and dynamical Coulomb blockade.

When the superconducting island is coupled to the bulk superconductor, we show that the current noise cross correlation is always negative as a function of dot energy levels at low lead chemical potentials. In contrast, the current cross correlation is always positive in a non-Majorana setup. For current cross correlations induced by the energy splitting of MBSs, a fourpeak cloverlike pattern of the noise cross correlation appears when tuning the dot energy levels. However, the pattern becomes more complex in the presence of a charging energy on the superconducting island. With the increase of lead chemical potentials, the cross correlation becomes positive gradually for the antisymmetric configuration of the dot energy levels. When the coupling strength between the island and the bulk superconductor is comparable to the charging energy, the noise cross correlation is strongly enhanced in the antisymmetric level configuration. The sign of the current cross correlation in the low lead chemical potentials can serve as a signature for the existence of the MBSs.

\section{ACKNOWLEDGMENTS}

This work was supported by the Research Grant Council, University Grants Committee, Hong Kong under Grant No. HKU703713P and the Natural Science Foundation of China under Grant No. 61474018 and No. 11574127.
[1] M. Z. Hasan and C. L. Kane, Rev. Mod. Phys. 82, 3045 (2010).

[2] X. L. Qi and S. C. Zhang, Rev. Mod. Phys. 83, 1057 (2011).

[3] S. Q. Shen, Topological Insulators: Dirac Equation in Condensed Matters (Springer, Berlin Heidelberg, 2012).

[4] J. D. Sau, R. M. Lutchyn, S. Tewari, and S. Das Sarma, Phys. Rev. Lett. 104, 040502 (2010).

[5] Y. Oreg, G. Refael, and F. von Oppen, Phys. Rev. Lett. 105, 177002 (2010).

[6] R. M. Lutchyn, J. D. Sau, and S. Das Sarma, Phys. Rev. Lett. 105, 077001 (2010).

[7] J. Alicea, Phys. Rev. B 81, 125318 (2010).

[8] J. D. Sau, S. Tewari, R. M. Lutchyn, T. D. Stanescu, and S. Das Sarma, Phys. Rev. B 82, 214509 (2010).

[9] C. W. J. Beenakker, Annu. Rev. Condens. Matter Phys. 4, 113 (2013).

[10] C. Nayak, S. H. Simon, A. Stern, M. Freedman, S. Das Sarma, Rev. Mod. Phys. 80, 1083 (2008).

[11] A. Stern, Nature (London) 464, 187 (2010).

[12] D. A. Ivanov, Phys. Rev. Lett. 86, 268 (2001).

[13] A. Kitaev, Ann. Phys. (NY) 303, 2 (2003).

[14] G. Moore and N. Read, Nucl. Phys. B 360, 362 (1991).

[15] L. Fu, Phys. Rev. Lett. 104, 056402 (2010).

[16] K. Flensberg, Phys. Rev. Lett. 106, 090503 (2011).

[17] M. Leijnse and K. Flensberg, Phys. Rev. Lett. 107, 210502 (2011).

[18] J. Alicea, Y. Oreg, G. Refael, F. von Oppen, and M. P. A. Fisher, Nat. Phys. 7, 412 (2011).

[19] L. Jiang, C. L. Kane, and J. Preskill, Phys. Rev. Lett. 106, 130504 (2011).

[20] P. Bonderson and R. M. Lutchyn, Phys. Rev. Lett. 106, 130505 (2011).

[21] V. Mourik, K. Zuo, S. M. Frolov, S. R. Plissard, E. P. A. M. Bakkers, and L. P. Kouwenhoven, Science 336, 1003 (2012).

[22] M. T. Deng, C. L. Yu, G. Y. Huang, M. Larsson, P. Caroff, and H. Q. Xu, Nano Lett. 12, 6414 (2012).

[23] A. Das, Y. Ronen, Y. Most, Y. Oreg, M. Heiblum, and H. Shtrikman, Nat. Phys. 8, 887 (2012).

[24] L. P. Rokhinson, X. Liu, and J. K. Furdyna, Nat. Phys. 8, 795 (2012).

[25] A. D. K. Finck, D. J. Van Harlingen, P. K. Mohseni, K. Jung, and X. Li, Phys. Rev. Lett. 110, 126406 (2013).
[26] S. Nadj-Perge, I. K. Drozdov, J. Li, H. Chen, S. Jeon, J. Seo, A. H. MacDonald, B. A. Bernevig, and A. Yazdani, Science 346, 602 (2014).

[27] D. Bagrets and A. Altland, Phys. Rev. Lett. 109, 227005 (2012).

[28] D. Pikulin, J. Dahlhaus, M. Wimmer, H. Schomerus, and C. Beenakker, New J. Phys. 14, 125011 (2012).

[29] J. Liu, A. C. Potter, K. T. Law, and P. A. Lee, Phys. Rev. Lett. 109, 267002 (2012).

[30] G. Kells, D. Meidan, and P. W. Brouwer, Phys. Rev. B 86, 100503 (2012).

[31] D. Rainis, L. Trifunovic, J. Klinovaja, and D. Loss, Phys. Rev. B 87, 024515 (2013).

[32] L. Hofstetter, S. Csonka, J. Nygård, and C. Schönenberger, Nature (London) 461, 960 (2009).

[33] L. Hofstetter, S. Csonka, A. Baumgartner, G. Fülöp, S d'Hollosy, J. Nygård, and C. Schönenberger, Phys. Rev. Lett. 107, 136801 (2011)

[34] J. Wei and V. Chandrasekhar, Nat. Phys. 6, 494 (2010).

[35] A. Das, Y. Ronen, M. Heiblum, D. Mahalu, A. V. Kretinin, and H. Shtrikman, Nat. Commun. 3, 1165 (2012).

[36] C. J. Bolech and E. Demler, Phys. Rev. Lett. 98, 237002 (2007).

[37] J. Nilsson, A. R. Akhmerov, and C. W. J. Beenakker, Phys. Rev. Lett. 101, 120403 (2008).

[38] S. Tewari, C. Zhang, S. Das Sarma, C. Nayak, and D. H. Lee, Phys. Rev. Lett. 100, 027001 (2008).

[39] K. T. Law, P. A. Lee, and T. K. Ng, Phys. Rev. Lett. 103, 237001 (2009).

[40] H. F. Lü, H. Z. Lu, and S. Q. Shen, Phys. Rev. B 86, 075318 (2012); 88, 159908(E) (2013).

[41] B. Zocher and B. Rosenow, Phys. Rev. Lett. 111, 036802 (2013).

[42] J. Liu, F. C. Zhang, and K. T. Law, Phys. Rev. B 88, 064509 (2013).

[43] Z. Wang, X. Y. Hu, Q. F. Liang, and X. Hu, Phys. Rev. B 87, 214513 (2013).

[44] P. Wang, Y. Cao, M. Gong, G. Xiong, and X. Q. Li, Europhys. Lett. 103, 57016 (2013).

[45] H. F. Lü, H. Z. Lu, and S. Q. Shen, Phys. Rev. B 90, 195404 (2014).

[46] S. Das Sarma, J. D. Sau, and T. D. Stanescu, Phys. Rev. B 86, 220506 (2012). 
[47] D. T. McClure, L. DiCarlo, Y. Zhang, H. A. Engel, C. M. Marcus, M. P. Hanson, and A. C. Gossard, Phys. Rev. Lett. 98, 056801 (2007).

[48] Y. Zhang, L. DiCarlo, D. T. McClure, M. Yamamoto, S. Tarucha, C. M. Marcus, M. P. Hanson, and A. C. Gossard, Phys. Rev. Lett. 99, 036603 (2007).

[49] S. Gangadharaiah, B. Braunecker, P. Simon, and D. Loss, Phys. Rev. Lett. 107, 036801 (2011).

[50] E. M. Stoudenmire, J. Alicea, O. A. Starykh, and M. P. A. Fisher, Phys. Rev. B 84, 014503 (2011).

[51] R. Hützen, A. Zazunov, B. Braunecker, A. L. Yeyati, and R. Egger, Phys. Rev. Lett. 109, 166403 (2012).

[52] A. Altland and R. Egger, Phys. Rev. Lett. 110, 196401 (2013).

[53] B. Béri and N. R. Cooper, Phys. Rev. Lett. 109, 156803 (2012).

[54] B. Béri, Phys. Rev. Lett. 110, 216803 (2013); M. R. Galpin, A. K. Mitchell, J. Temaismithi, D. E. Logan, B. Béri, and N. R. Cooper, Phys. Rev. B 89, 045143 (2014).

[55] J. Ulrich and F. Hassler, Phys. Rev. B 92, 075443 (2015).

[56] M. A. Sillanpää, T. Lehtinen, A. Paila, Yu. Makhlin, L. Roschier, and P. J. Hakonen, Phys. Rev. Lett. 95, 206806 (2005).

[57] O. Naaman and J. Aumentado, Phys. Rev. Lett. 98, 227001 (2007).

[58] A. Zazunov, A. L. Yeyati, and R. Egger, Phys. Rev. B 84, 165440 (2011).

[59] A. Zazunov and R. Egger, Phys. Rev. B 85, 104514 (2012).

[60] S. Plugge, A. Zazunov, P. Sodano, and R. Egger, Phys. Rev. B 91, 214507 (2015).

[61] H. Bruus and K. Flensberg, Many-Body Quantum Theory in Condensed Matter Physics (Oxford University Press, Oxford, UK, 2004).
[62] V. Kashcheyevs, A. Aharony, and O. Entin-Wohlman, Phys. Rev. B 73, 125338 (2006).

[63] A. Mitra and A. J. Millis, Phys. Rev. B 76, 085342 (2007).

[64] C. Timm, Phys. Rev. B 77, 195416 (2008).

[65] C. Pöltl, C. Emary, and T. Brandes, Phys. Rev. B 80, 115313 (2009).

[66] H. Z. Lu, B. Zhou, and S. Q. Shen, Phys. Rev. B 79, 174419 (2009).

[67] A. Cottet, W. Belzig, and C. Bruder, Phys. Rev. Lett. 92, 206801 (2004).

[68] J. Wabnig, B. W. Lovett, J. H. Jefferson, and G. A. D. Briggs, Phys. Rev. Lett. 102, 016802 (2009).

[69] D. Dubrovin and E. Eisenberg, Phys. Rev. B 76, 195330 (2007).

[70] A. Thielmann, M. H. Hettler, J. König, and G. Schön, Phys. Rev. B 71, 045341 (2005).

[71] J. Aghassi, A. Thielmann, M. H. Hettler, and G. Schön, Phys. Rev. B 73, 195323 (2006).

[72] F. Elste and C. Timm, Phys. Rev. B 73, 235305 (2006).

[73] I. Weymann, Phys. Rev. B 78, 045310 (2008).

[74] I. Weymann, B. R. Bułka, and J. Barnaś, Phys. Rev. B 83, 195302 (2011).

[75] J. Koch and F. von Oppen, Phys. Rev. Lett. 94, 206804 (2005).

[76] H. Hübener and T. Brandes, Phys. Rev. Lett. 99, 247206 (2007).

[77] J. Eldridge, M. G. Pala, M. Governale, and J. König, Phys. Rev. B 82, 184507 (2010).

[78] M. Governale, M. G. Pala, and J. König, Phys. Rev. B 77, 134513 (2008).

[79] M. Leijnse and K. Flensberg, Phys. Rev. B 86, 134528 (2012). 